\title{
EVOLUTIONARY COMPUTING FOR METALS PROPERTIES MODELLING
}

\author{
M.F. Abbod*, M. Mahfouf, D.A. Linkens and Sellars, C.M.
}

\section{IMMPETUS}

Institute for Microstructure and Mechanical Properties Engineering, The University of Sheffield

Department of Automatic Control and Systems Engineering,

The University of Sheffield, Sheffield, UK

*Email:M.F.Abbod@shef.ac.uk

\begin{abstract}
During the last decade Genetic Programming (GP) has emerged as an efficient methodology for teaching computers how to program themselves. This paper presents research work which utilises GP for developing mathematical equations for the response surfaces that have been generated using hybrid modelling techniques for predicting the properties of hot deformation of materials. Collected data from the literature and experimental work on aluminium are utilised as the initial training data for the GP to develop the mathematical models under different deformation conditions and compositions.
\end{abstract}

Keywords: Alloy materials, genetic programming, material properties, modelling, strain, stress.

\section{INTRODUCTION}

One of the central challenges of computer science is to get a computer to perform certain tasks without instructing it how to do it. Genetic programming addresses this challenge by providing a method for automatically creating a working computer program from a high-level statement of the problem.

Genetic Programming (GP), invented by Cramer in 1985 and further developed by Koza (1992), solved the problem of fixed length solutions by creating non-linear entities with different sizes and shapes. The repertoire used to create these entities was also more varied, creating a richer, more versatile system of representation.

GP achieves this goal of automatic programming (also sometimes called program synthesis or program induction) by genetically breeding a population of computer programs using the principles of Darwinian natural selection and biologically inspired operations. The operations include reproduction, crossover (sexual recombination), mutation, and architecture-altering operations patterned after gene duplication and gene deletion in nature.

This paper is based on developing mathematical models for hybrid models that have been developed for modelling metals properties under hot deformation conditions. Collected data from the literature and experimental work for aluminium alloys are utilised as the databases for the models. Under different deformation conditions and compositions, data points are available for the flow stress and recrystallisation.

\section{GENETIC PROGRAMMING}

Genetic programming starts with a primordial large set of randomly-generated computer programs. The set of functions that may appear at the internal points of a program tree may include ordinary arithmetic functions and conditional operators. The set of terminals appearing at the external points typically include the program's external inputs (such as the independent variables $\mathrm{X}$ and $\mathrm{Y}$ ) and random constants (such as 3.2 and 0.4). The randomly created programs typically have different sizes and shapes

Consider, for example, the following algebraic expression: 


$$
\frac{a \times b}{c}+\sqrt{d-e}
$$

It can also be represented as a diagram which is shown in Figure 1.

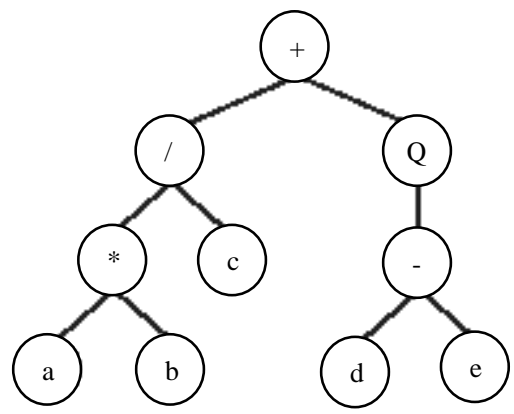

Fig. 1. Genetic programming tree.

where 'Q' represents the square root function. This kind of diagram representation is in fact the phenotype of GP chromosomes, being the genotype easily inferred from the phenotype as follows:

0123456789

$+/ \mathrm{Q} *$ c-abde

which is the straightforward reading of the equation from left to right and from top to bottom (exactly as ones read a page of text). The expression is an Open Reading Frame, starting at ' + ' (position 0 ) and terminating at ' $e$ ' (position 9). The subtraction and division are functions of two arguments, and therefore in the next line are placed four more nodes, in this case, the symbols at positions 3, 4, 5, and 6. Now, we have two different functions in the third line: one is a function of one argument $(\mathrm{Q})$, and another a function of two arguments $(+)$. Therefore, three more nodes are required in the next line. In this case, they are filled with the elements at positions 7,8 , and 9 .

\subsection{Solving Problems with Genetic Programming}

The aim of this study is to utilise GP for finding mathematical equations as a solution for modelling the behaviour of metals based on specified input conditions.

In symbolic regression or function-finding the goal is to find an expression that satisfactorily explains the dependent variable. The input into the system is a set of fitness cases in the form $\left(a_{(i, 0)}, a_{(i, 1)}, \ldots, a_{(i, \mathrm{n}-1)}, y_{i}\right)$ where $a_{(i, 0)}-a_{(i, \mathrm{n}-1)}$ are the independent variables and $y_{i}$ is the dependent variable. The set of fitness cases consists of the adaptation environment where solutions adapt, thus discovering, solutions to problems.

There are five major steps in preparing to use GP. The first is to choose the fitness function. For instance, we could measure the fitness $f_{i}$ of an individual program $i$ by the following expression:

$$
f_{i}=\sum_{j=1}^{c_{t}}\left(M-\left|C_{(i, j)}-T_{j}\right|\right.
$$

where $M$ is the range of selection, $C_{(i, j)}$ the value returned by the individual chromosome $i$ for fitness case $j$ (out of $C_{t}$ fitness cases), and $T_{j}$ is the target value for fitness case $j$. If $\left|C_{(i, j)}-T_{j}\right|$ (the precision) is less than or equal to 0.01 , then the precision is equal to zero, and $f_{i}=f_{\max }=C_{t} . M$. (Ferreira 2001).

The second major step consists of choosing a set of terminals $T$ and a set of functions $F$ to create the chromosomes. The third major step is to choose the chromosomal architecture, i.e., the length of the individual and the number of genes. The fourth major step in preparing to use GP is to choose the linking function. Finally, the fifth major step is to choose the set of genetic operators that cause variation and their rates. 


\section{MATERIAL PROPERTIES MODELLING}

One method for modelling of metals properties is the hybrid modelling technique which provides a good modelling tool which combines different modelling approaches in one dynamic model (Abbod et al, 2002). Combinations of physical models and neuro-fuzzy models work very efficiently. While the physical model ensures that the results are physically sensible, the neuro-fuzzy model can be utilised to increase the accuracy of the results. One of the possible hybrid modelling techniques is the semi-parametric model which utilises serial and parallel parametric and non-parametric models. This gives advantages for compensating for sparse data, extrapolation improvements, uncertainty and the bias of the default model.

The ultimate aim of modelling the metals behaviour, however, is to be able to provide tools that can be extended to all conditions and for other material. Currently, physically-based modelling is difficult to apply to extended conditions and materials. To extend the region of application requires the carrying out of a huge amount of experimental research, which is a very time-consuming process. Therefore, it is desirable to develop a modelling tool capable of being used for prediction under extended deformation conditions and for other kinds of metals.

The hybrid modelling is carried out by combining the developed neuro-fuzzy models to predict the internal states, while the physically-based models all used to predict the final recrystallisation and flow stress conditions (Zhu et al, 2003). In the hybrid model, the inputs are the deformation conditions such as strain rate, temperature $T$ and deformation level, i.e. strain for a given material. The friction stress is at present difficult to calculate theoretically and hence is calculated using a neuro-fuzzy model based on experimental results. The internal state variables such as internal dislocation density $\rho_{l}$, subgrain size $\delta$ and misorientation between subgrains $\theta$ are calculated by neuro-fuzzy models. The neuro-fuzzy models were trained based on data extracted from fitted smoothed curves of the original experimental measurements. Then, using the physical equations the final flow stress and the recrystallisation behaviour $\left(\mathrm{N}_{\mathrm{v}}, \mathrm{d}_{\mathrm{rex}}, \mathrm{t}_{50}\right)$ are determined. Since the model covers a wide range of temperature and strain rate for each individual composition, tuning the model was important to adjust some of the parameters for scaling the internal state variables and calculating the individual internal stresses.

\section{FLOW STRESS MODELLING}

The generalisation of the model to a wide range of deformation conditions and compositions started with collecting data from the literature for different authors and different deformation conditions. The validation process of the hybrid model is based on the input-output relationships, in other words the input deformation conditions, composition, and the output flow stress and the recrystallisation kinetics. The intermediate variables (internal states) are validated based on the output of the model using the physical equations.

\subsection{Model Developments}

Model prediction has been performed for different alloys based on the experimental results from different researchers. It was observed that the most important elements which have a significant effect on the flow stress are $\mathrm{Mg}, \mathrm{Mn}$ and Fe. Therefore, in order to develop a global model, the effects of the alloying elements have to be considered as the major inputs to the composition block. In designing experimental conditions for developing such a model, the input parameters to be considered are the strain, strain rate, temperature, and composition $(\mathrm{Mg}$, and other alloying elements).

The collected data from the literature were based on the input deformation conditions, the composition of the material, and deformation conditions, and any other related information which might be of use in the future in order to explain some of the unexpected behaviour that might occur during the modelling stage. Information of particular importance is the composition of the material which has been recorded for most of the collected data.

One of the important factors that affect the final properties of the metals is the level of impurities. During the validation process it was noted that for commercial alloys, the impurities might be very high such that they exceed the solubility limits. Therefore, coarse second phase particles are formed which do not affect the final flow stress of the material. Therefore, it was important to test the limits of the solubility of the impurities because it is considered to be an important factor for the model prediction.

The developed hybrid model is based on commercial purity $\mathrm{Al}-1 \% \mathrm{Mg}$ alloy which has some impurities, therefore it is expected that for high purity aluminium the predicted flow stress should be higher than the actual values. The same statement is valid for commercial purity aluminium, as the inclusion of more impurities should increase the flow stress while the hybrid model will under-predict the flow stress. For commercial alloys, the 
model under-predicts the flow stress due to the effect of the magnesium whose presence increases the flow stress.

Using the calculated slopes for different compositions, the final effect of the impurities on the slope of the predicted flow stress behaviour is divided into two steps; for pure aluminium, and for commercial aluminium. The two relationships for the pure aluminium and aluminium alloys can be combined together to generate a 3D surface that represents the effects of the impurities and $\mathrm{Mg}$ content on the final predicted flow stress of the material.

The dynamic evolution of the flow stress should be based on some parameters, which are the initial flow stress, the final flow stress and the strain when steady-state conditions are reached. The dynamic behaviour of the flow stress is governed by the following equations:

$$
\begin{aligned}
& \varepsilon_{r}=a+b \sigma_{s s}^{2} \\
& \sigma_{0}=c \sigma_{s s}
\end{aligned}
$$

where $a, b$ and c should be composition-dependant only.

The final flow stress can be calculated using the following equation:

$$
\sigma=\sigma_{0}+\left(\sigma_{s s}-\sigma_{0}\right)\left[\left(1-\exp \left(\frac{-\varepsilon}{\varepsilon_{r}}\right)\right)\right]^{1 / 2}
$$

The start of steady-state $\varepsilon_{\mathrm{s}}$ is defined as follows:

$$
\left(\frac{\sigma-\sigma_{0}}{\sigma_{s s}-\sigma_{0}}\right)=0.95 ; \text { then } \varepsilon_{s s}=3.2 \varepsilon_{r}
$$

A graphical representation of the flow stress curve behaviour is shown in figure 2.

Table 1 shows the data collected from the literature via different authors. All the related information is listed in this table.

The initial values for the flow stress are not easy to quantify. This is because the figures are digitally quantised from different sources, therefore, it was not always clear what the initial values were. Since these locations are not easy to distinguish and quantify, it has been decided to select the steady-state flow stress $\left(\sigma_{\mathrm{ss}}\right)$, the relaxation stress $\left(\sigma_{\mathrm{r}}\right)$ and the relaxation strain $\left(\varepsilon_{\mathrm{r}}\right)$.

Using the collected and quatitised data, three 3-D surfaces were generated which describe the relationship between the $\mathrm{Mg}$ contents, the impurities and the steady-state flow stress $\left(\sigma_{\mathrm{ss}}\right)$, relaxation stress $\left(\sigma_{\mathrm{r}}\right)$ and relaxation strain $\left(\varepsilon_{\mathrm{r}}\right)$ as shown in Figure 3-5(a) respectively. The surfaces were generated using a neuro-fuzzy modelling technique.

\section{MODELLING RESULTS}

One important feature of GP is that the initial structure of the equations can be predefined and constrained for both the parameters limits and the algebraic formulas. With the initial metallurgical knowledge of such formulation, the equations can be predefined and formulated to evolve to semi-physical equations. Different input variables can be used such as the deformation conditions and the composition of the material.

The developed 3-D surfaces which describe the relationship between the impurities, $\mathrm{Mg}$ contents and the predicted slope are based on neuro-fuzzy models which are data-driven. Such models are linguistically interpretable in terms of the input and output variables. Nevertheless, a mathematical equation can be used to model the surfaces based on the data generated by the neuro-fuzzy models. The modelling of such surfaces requires knowledge of the basic components of a polynomial equation. The structure of the polynomial equation can be made flexible to select the best fit that minimises the error between the predicted and modelled surfaces. GP is one method which allows such flexibility in the structure. 
Typically, an initial function structure can be based on the basic mathematical operators (+-/*) and the input variables, with some knowledge being available about the sort of surface. Some functions are supplied to the algorithm to start as an initial programming seed. Such functions are $\exp$ (impurities), $M g^{3}, M g^{2}$, and $M g$.

The predicted equations for the steady state flow stress and $\varepsilon_{\mathrm{r}}$ were found to be respectively:

$$
\begin{aligned}
& \text { stress }_{s s}=G\left(1-\exp \left(\frac{-i m}{T}\right)+A M g^{3}+B M g^{2}+C M g+D\right. \\
& +\frac{M g}{64\left(M g^{6}-9\right) i m} \\
& \text { stress }_{r}=G\left(1-\exp \left(\frac{-i m}{T}\right)\right)+A M g^{3}+B M g^{2}+C M g \\
& +D-1 \\
& \text { strain }_{r}=G M g\left(1-R \exp \left(\frac{-i m}{T}\right)\right)+A M g^{3}+B M g^{2}+C M g \\
& +D+\frac{M g^{2}}{2}
\end{aligned}
$$

where im denotes the impurities. The equations parameters are as shown in Table 2. Figures 3-5(b) show the prediction accuracy for the flow stress, relaxation stress and relaxation strain respectively.

\begin{tabular}{|l|c|c|c|}
\hline parameters & stress_ss & stress_r & strain_rr \\
\hline G & 0.55696 & 0.53517 & 0.55777 \\
\hline R & & & 0.66129 \\
\hline T & 0.05033 & 0.04879 & 0.05196 \\
\hline A & -0.01666 & -0.02315 & -0.05088 \\
\hline B & 0.08998 & 0.12599 & 0.37836 \\
\hline C & 0.22410 & 0.30580 & -0.56219 \\
\hline D & 0.28571 & 0.60565 & 0.74449 \\
\hline error & 0.00056 & 0.00158 & 0.00146 \\
\hline
\end{tabular}

Table 2: GP parameters fitting.

\section{CONCLUSIONS}

The developed hybrid models have been extensively validated against different experimental results from different authors and different deformation conditions. The developed model can be used by both the research community and industry as it provides the internal states as well as the final metals properties. Furthermore, GP was utilised to generate mathematical models for a wide range of compositions that can be used to predict the material properties without the need for experimental testing which may prove to be an expensive and time consuming exercise.

\section{ACKNOWLEDGMENT}

The authors gratefully acknowledge the UK EPSRC (Engineering and Physical Sciences Research Council) for their financial support under grant number GR/R70514/01. 


\section{REFERENCES}

Abbod, M.F., D.A. Linkens and Q. Zhu (2002). Semi-physical modelling of internal states for aluminium alloys. Materials Science and Technology, vol A333, pp 397-408.

Cramer, N.L. (1985). A representation for the adaptive generation of simple sequential programs. In J. J. Grefenstette, ed., Proceedings of the First International Conference on Genetic Algorithms and Their Applications, Erlbaum.

Ferreira, C. (2001). Gene expression programming: a new adaptive algorithm for solving problems. Complex Systems.

Koza, J.R. (1992). Genetic Programming: On the Programming of Computers by Means of Natural Selection, Cambridge, MA: MIT Press.

Zhu, Q., M.F. Abbod, J. Talamantes-Silva, C.M. Sellars, D.A. Linkens and J.H. Beynon (2003). Hybrid modelling of aluminium-magnesium alloys during thermomechanical processing in terms of physicallybased, neuro-fuzzy and finite elements models", Acta Mat, (in press).

\begin{tabular}{|l|c|c|c|c|c|}
\hline \multicolumn{1}{|c|}{ material } & Mg $(\boldsymbol{\%})$ & Impurities & $\sigma_{\mathbf{r}}(\mathbf{M P a})$ & $\sigma_{\text {ss }}(\mathbf{M P a})$ & $\varepsilon_{\mathbf{r}}$ \\
\hline $\mathrm{CP} \mathrm{Al}$ & 0.00 & 0.0000 & 1.5988 & 2.0376 & 1.0609 \\
\hline $\mathrm{AL} 99.997$ & 0.00 & 0.0000 & 1.9323 & 3.0671 & 0.8708 \\
\hline $\mathrm{Al}$ & 0.0011 & 0.0045 & 1.2278 & 1.6010 & 1.5992 \\
\hline $\mathrm{Al}-1 \mathrm{Mn}$ & 0.016 & 1.0154 & 0.8774 & 1.2176 & 1.3940 \\
\hline $\mathrm{Al}-0.5 \mathrm{Mg}$ & 0.50 & 0.0000 & 1.1125 & 1.5599 & 1.1818 \\
\hline $\mathrm{Al}-1 \mathrm{Mg}$ & 0.94 & 0.0031 & 1.0000 & 1.0000 & 1.0000 \\
\hline $\mathrm{Al}-1 \mathrm{Mg}$ & 0.94 & 0.0031 & 0.6190 & 1.4842 & 1.3666 \\
\hline $\mathrm{Al}-0.96 \mathrm{Mg}$ & 0.96 & 0.0000 & 0.8194 & 1.2623 & 1.2000 \\
\hline $\mathrm{Al}-\mathrm{Mg}$ & 1.00 & 0.0000 & 0.6837 & 0.9508 & 0.7683 \\
\hline $\mathrm{Al}-\mathrm{Mg}-\mathrm{Mn}$ & 1.09 & 1.0040 & 0.4681 & 0.6457 & 0.5000 \\
\hline $\mathrm{AA} 5052$ & 2.70 & 0.0620 & 0.3571 & 0.4797 & 0.3373 \\
\hline
\end{tabular}

Table 1: Calculated parameters for the developed correction surfaces.

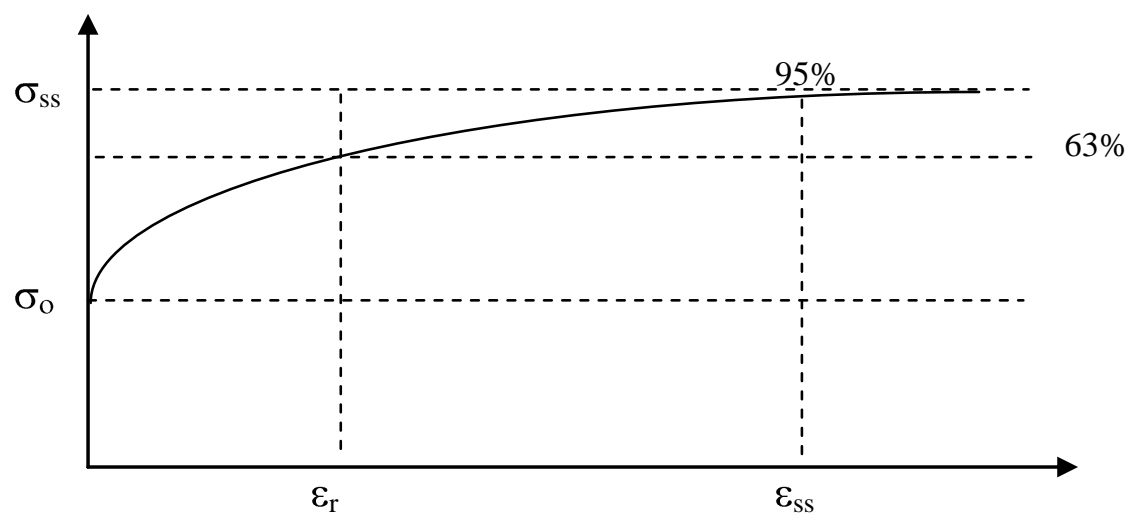

Fig. 2. Graphical presentation of the dynamic behaviour of the flow stress. 


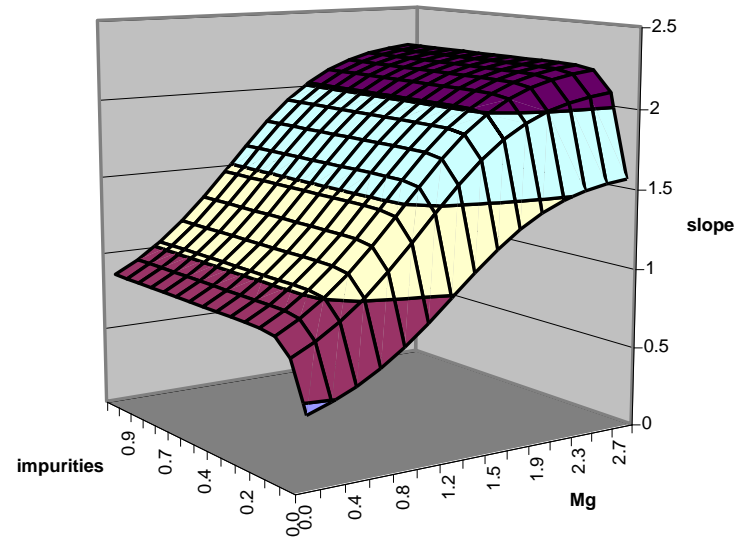

(a) NF response surface

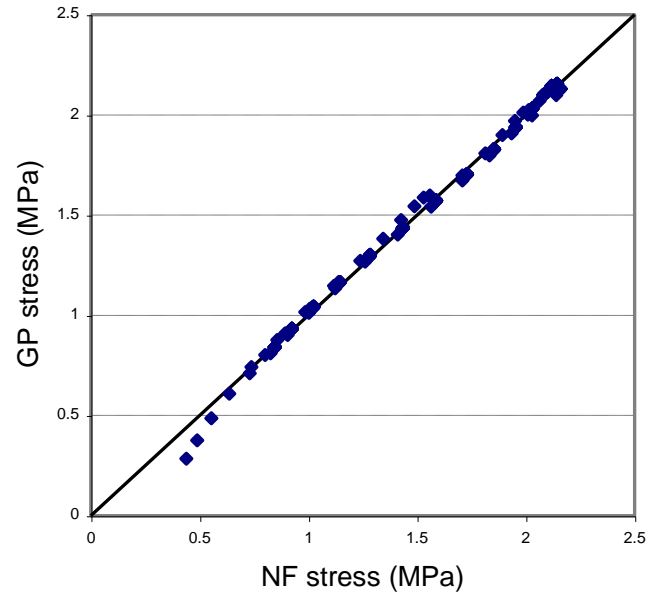

(b) GP equation prediction

Fig. 3. Steady-state flow stress modelling.

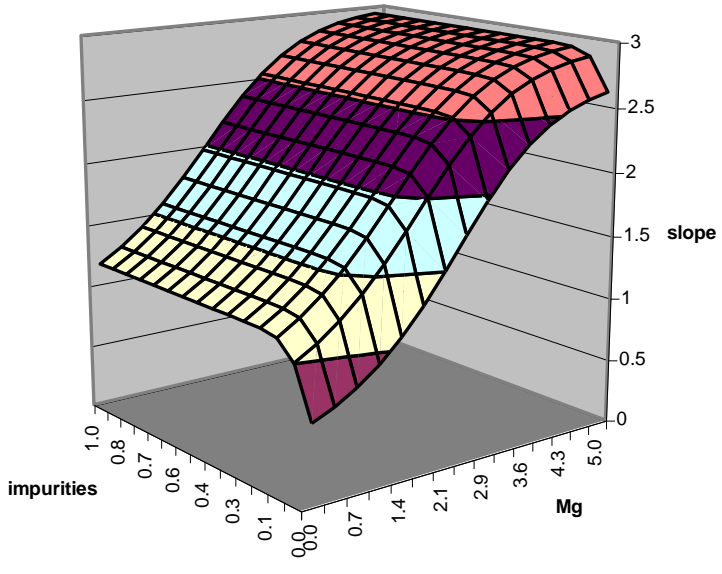

(a) NF response surface

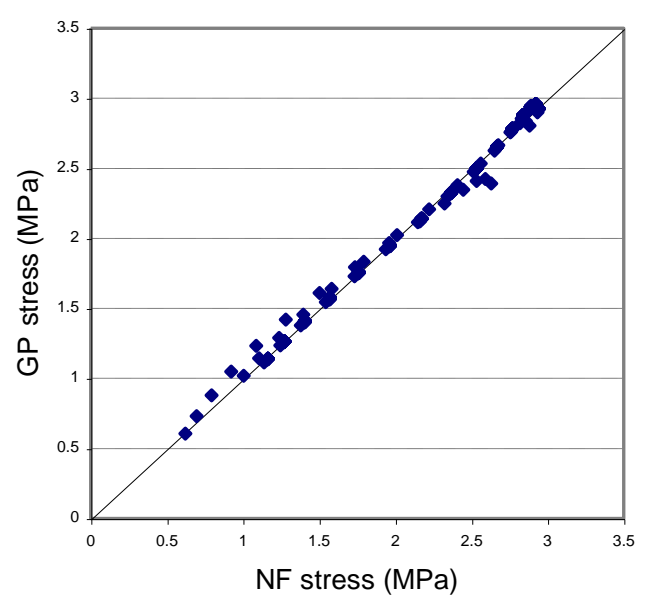

(b) GP equation prediction

Fig. 4. Relaxation stress modelling. 


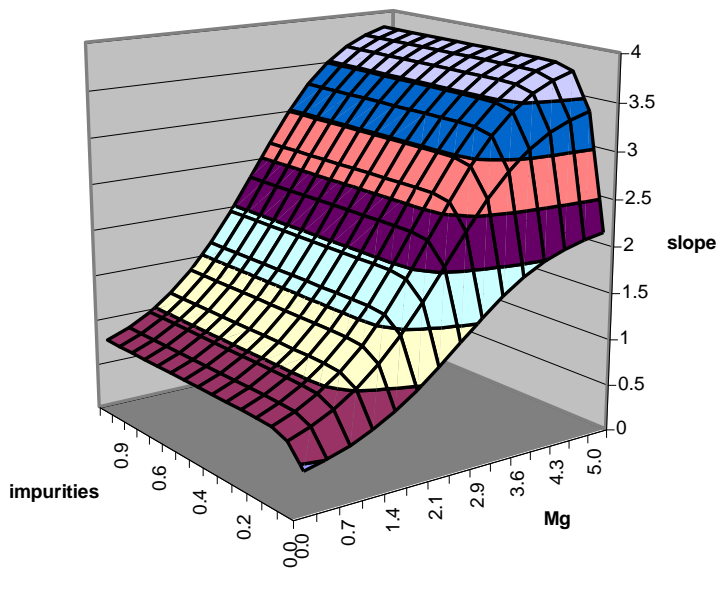

(a) NF response surface

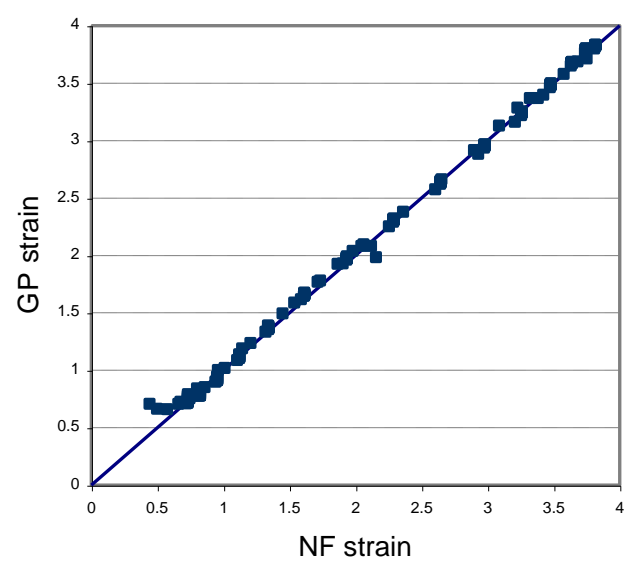

(b) GP equation prediction

Fig. 5. Relaxation strain modelling. 